\title{
Traditional Chinese Medicine and Its Significance
}

Fasi Ahamad $\mathbf{S}^{1^{*}}$ Jhansi $\mathrm{K}^{2}$ and Satya Varali ${ }^{3}$

${ }^{1}$ Department of Life sciences, University of Wolverhampton, UK

${ }^{2}$ Department of Biochemistry, Dr. L.B. College, Andhra University, Visakhapatnam, India

${ }^{3}$ Department of Human Genetics, Andhra University, Visakhapatnam, India

*Corresponding author: Fasi Ahamad S, Department of Life sciences, University of Wolverhampton, UK, Tel: 91-7842466596; E-mail: fasi.ahamed@gmail.com Rec date: Feb 15, 2015, Acc date: Feb 20, 2015, Pub date: Feb 23, 2015

Copyright: (c) 2015 Fasi AS, et al. This is an open-access article distributed under the terms of the Creative Commons Attribution License, which permits unrestricted use, distribution, and reproduction in any medium, provided the original author and source are credited.

\begin{abstract}
In this review, traditional Chinese medicine (TCM) history and importance were discussed. Tools and methods in the therapy were acknowledged. By TCM therapy treatment using herbal products, we can insert the energy into meridians and muscles which increase the person's life force.
\end{abstract}

Keywords Traditional Chinese medicine; Diagnosis; Treatment; Drug discovery

\section{History}

Traditional Chinese medicine has been using more than 2000 years and becoming popular in recent years across the world. It is a unique system therapy to diagnose and cure the diseases. Herbal medicine products are using in drug discovery, dietary and functional food elements [4].

\section{Introduction}

Natural products like plant herbs, fruits and flowers have been using for the alternative treatment in the medicine. Traditional medicine practitioners have been using these products as antigenic agents for the treatment. Now days by using traditional Chinese medicine, many diseases are treating with unique advantages and unremarkable effects. By using some Chinese herbs pancreas, stomach and intestine related diseases can be treated [1-2].

Traditional Chinese medicine is one of the acknowledged natural medicines classified by world health organization. It can be treated for many diseases. Traditional Chinese medicine consists of natural plant herbs, herbal products and also may contain non-plant substances [3]. Traditional Chinese medicine is a concept of Qi which means vital energy. Qi acts as energy carrier during Acupuncture. Traditional Chinese medicine treatment primarily focuses on entire system and then it corrects the pathological changes of the organ [4].

\section{Tools in diagnosis}

The theory of five elements i.e wood, fire, earth, metal and water was used to interrupt the relation between pathology and physiology of human body. Some organs and tissues in the body are having similar properties with these five elements as those five elements interact pathologically and physiologically. These five elements promote each other as wood promotes fire, fire promotes earth, earth promotes metal, metal promotes water and water promotes and generates wood. This circle is endless [5].
For diagnosis of diseases there are some tools essential for the treatment. They are diagnosis energy, Chinese pharmacology, acupuncture, moxibustion, cupping and massage [6].

\section{Diagnosis energy:}

In this part, diagnostic points will be examined example: eyes, tongue, wrest and skin

\section{Chinese pharmacology}

In this part plants, animals and minerals products are applicable in different ways

\section{Acupuncture}

Introducing thin needles into specific points of the body where the energy can be flows. It is generally considered as safety treatment when it was performed by experienced practitioner. The practice of acupuncture therapy is based on meridians theory. Through meridians, energy and blood circulates in the body.

\section{Herbal Therapy}

Herbal medicine along with the acupuncture is a main pillar of traditional Chinese medicine. Medicinal herbs show effect on enhancing and activating metabolism of the body.

\section{Moxibustion}

Encouraging the acupuncture points with the heat of torch light Eg: mugwort.

\section{Cupping}

Applying cups on the patients skin after air insertion.

\section{Massage}

Applying on bones and joints, muscles, acupuncture and meridian points. 
Citation: Fasi AS, Jhansi K, Satya V (2015) Traditional Chinese Medicine and Its Significance. Med chem 5: 01-R. doi:

Page 2 of 2

\section{Advantages}

Unique applications are there for diagnosis and treatment. By using traditional Chinese medicine treatment, we not only prevent the diseases but also pay attention to the health care. Treatment will be done without using any drug. Many incurable, chronic and geriatric diseases can be treated. traditional Chinese medicine is safe, simple and inexpensive [7].

There will be no side effects in the treatment as in the western medicine, some diseases may occur because of antibiotic reactions. By TCM treatment, a person's energy (life force) can be increased and also energy can be easily moved into meridians and muscles [8].

\section{Conclusion}

There are more benefits by using traditional Chinese medicine therapies as discussed in the advantages part. Still, some implementation is needed in the treatment methodology.

\section{References}

1. Yang L, He W, Qu H, Jia C, Wang Y, et al. (2014) Phytochemical Isoliquiritigenin Inhibits Angiogenesis Ex Vivo and Corneal Neovascularization in Mice. Altern Integr Med 3:176. doi: 10.4172/2327-5162.1000176.

2. Xi-Ping Zhang, Yan Shi, Ling Zhang (2007) Progress in the Study of Therapeutic Effects of Traditional Chinese Medicine and Extracts in Treating Severe Acute Pancreatitis. J Pancreas 8:704-714.

3. Tam K, Banh HL (2014) Attitudes of Alberta Pharmacists Pertaining to Traditional Chinese Medicine Practice and Complementary Alternative Medicine. J Pharma Care Health Sys 1:108. doi: 10.4172/jpchs.1000108

4. Traditional Chinese Medicine Information

5. Zhong LLD, Hu DD, Xu HX, Han QB, Bian ZX (2014) Efficacy-Driven Quality Control Platform for Chinese Herbal Medicine. Pharm Anal Acta 5:291. doi: 10.4172/2153-2435.1000291.

6. Pelullo CP, Abuadili AV, Iommelli O, Attena F (2015) Self-reported Changes in Health Status in a Traditional Chinese Medicine Centre: An Observational Study. J Nov Physiother 5:243. doi: 10.4172/2165-7025.1000243

7. Advantages of Traditional Chinese Medicine

8. The Benefits of Traditional Chinese Medicine for Children 